\title{
High-efficiency Köhler photovoltaic concentrators with external light confinement
}

\author{
Pablo Zamora a , Pablo Benítez ${ }^{\mathrm{a}, \mathrm{b}}$, Juan C. Miñano ${ }^{\mathrm{a}, \mathrm{b}}$, Yang Li ${ }^{\mathrm{c}}$, Marina Buljan ${ }^{\mathrm{a}}$ \\ ${ }^{\text {a } C E D I N T, ~ T e c h n i c a l ~ U n i v e r s i t y ~ o f ~ M a d r i d ~(U P M), ~ C a m p u s ~ d e ~ M o n t e g a n c e d o ~} 28223$ Pozuelo de \\ Alarcón, Madrid, Spain \\ ${ }^{\mathrm{b}}$ LPI-LLC, 2400 Lincoln Ave., Altadena, CA 91001, USA \\ c Zhejiang University, 338 Zheda Road, Hangzhou 310027, China
}

\begin{abstract}
Metal grid lines are a vital element in multijunction solar cells in order to take out from the cell the generated photocurrent. Nevertheless all this implies certain shadowing factor and thus certain reflectivity on cells surface that lowers its light absorption. This reflectivity produces a loss in electrical efficiency and thus a loss in global energy production for CPV systems. We present here an optical design for recovering this portion of reflected light, and thus leading to a system efficiency increase. This new design is based on an external confinement cavity, an optical element able to redirect the light reflected by the cell towards its surface again. It has been possible thanks to the recent invention of the advanced Köhler concentrators by LPI, likely to integrate one of these cavities easily. We have proven the excellent performance of these cavities integrated in this kind of CPV modules offering outstanding results: $33.2 \%$ module electrical efficiency @Tcell=25 $5^{\circ} \mathrm{C}$ and relative efficiency and $I_{s c}$ gains of over 6\%.
\end{abstract}

Keywords: concentrator optics, confinement cavity, nonimaging optics

\section{INTRODUCTION}

Multijunction (MJ) cells reflectivity is mainly due to two different reasons: light reflection produced by grid lines and inherent reflectivity of the cell uncovered semiconductor surface. MJ semiconductor surfaces are always covered by an anti-reflective (AR) coating able to minimize light reflection on the cell surface. Even if reduced to the maximum, these two different absorptions will always be present in any solar cell. Our strategy to improve CPV modules performance is thus based on trying to recover this reflected light in order to increase light absorption in the cell and so global electrical efficiency. This work presents a solution to this problem based on an optical design including an external confinement cavity [1].

The recent invention of high-performance LPI Fresnel-Köhler concentrators (FK [2], FRXI [3] and Fresnel RXI-RR [4]), fully compatible with the use of these external cavities, have allowed for the practical integration of this structure inside a CPV module [3]. In this way a prototype with cavity has been manufactured, based on a FK (Fresnel-Köhler) architecture. We will present several results for this module: theoretical calculations and experimental (indoors and outdoors) measurements, showing all of them a neat efficiency gain due to the external cavity effect.

We have built up this FK module as a proof of concept of confinement cavities working in CPV concentrators. In this way we will show in the measurements chapter outstanding results attained by our FK-Cavity module. Integration of these cavities in more complex CPV modules is also possible, as shown in a promising paper, including spectrum splitting in the design and other innovative strategies as well [4].

\section{CELL REFLECTIVITY AND CAVITY DESIGN}

High concentration levels produce higher current densities as compared to one-sun situations (no concentration). In the case of multijunction solar cells, the front grid design must be optimized for the trade-off between series resistance losses and loss of absorbed light due to front metal grid shading factor $\left(f_{s}\right)$. In today's high concentration multijunction solar cells, $f_{s}$ presents values in the 8-12\% range. Regarding cell reflectivity, $f_{s}$ and AR coatings are optimized for the 400900nm wavelength range (corresponding to top and middle sub-cells range) but the reflection in the further infrared is higher. This does not affect multijunction cells performance because of the bottom sub-cell excess of current. 
Two different strategies have been used in the past to minimize this undesired reflection of useful light produced by the front metal grid. The first one consists in preventing the light from hitting the grid lines, for instance using refractive prismatic covers aligned over the grid lines [5]. However, this approach presents several disadvantages. It does not recover the light reflected on the semiconductor surface, needs very precise alignment, and is not effective under wideangle illumination (as occurs in high-concentration systems), especially when a secondary optical element (SOE) is optically coupled to the cell as a secondary concentrator or homogenizer. The second strategy consists on reflective covers, but their implementation also has practical challenges [6].

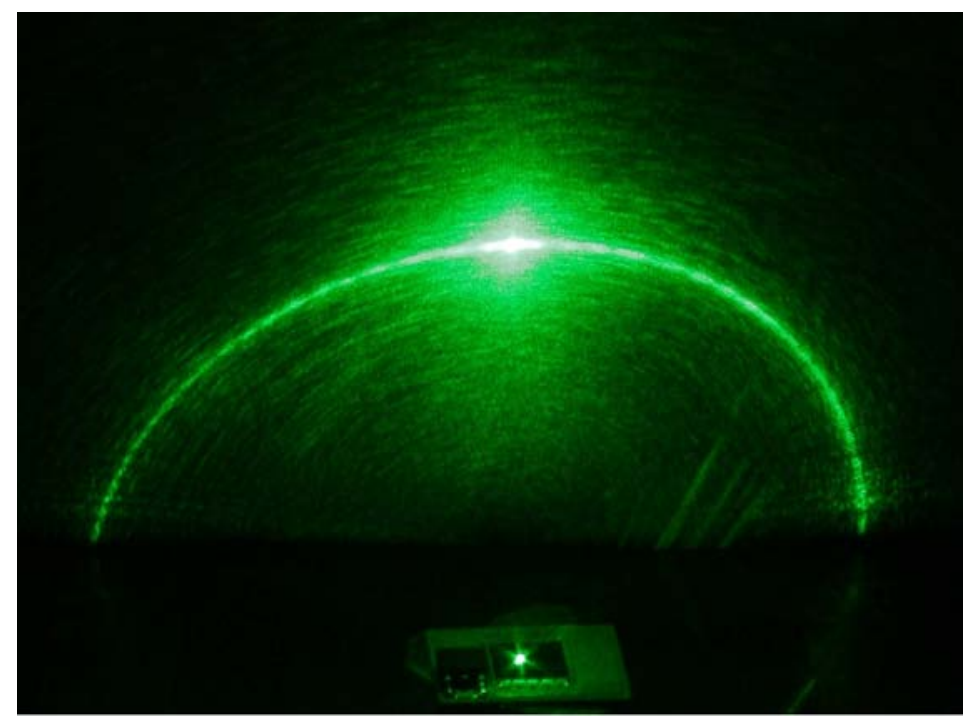

Figure 1 Solar cell reflection pattern. Light scattered on the surface of a vertical plane behind the cell. Cell is placed on the bottom part of the figure, with grid lines perpendicular to vertical plane.

On the other hand, a third strategy, referred to as external confinement [1], is more compatible with high concentration. It consists on using a refractive material cavity with reflective surface (achieved by metalizing it), that collects the light reflected by the cell (either by the grid lines or by the semiconductor surface) and sending it back to the cell. Besides, external confinement does not need precise alignment.
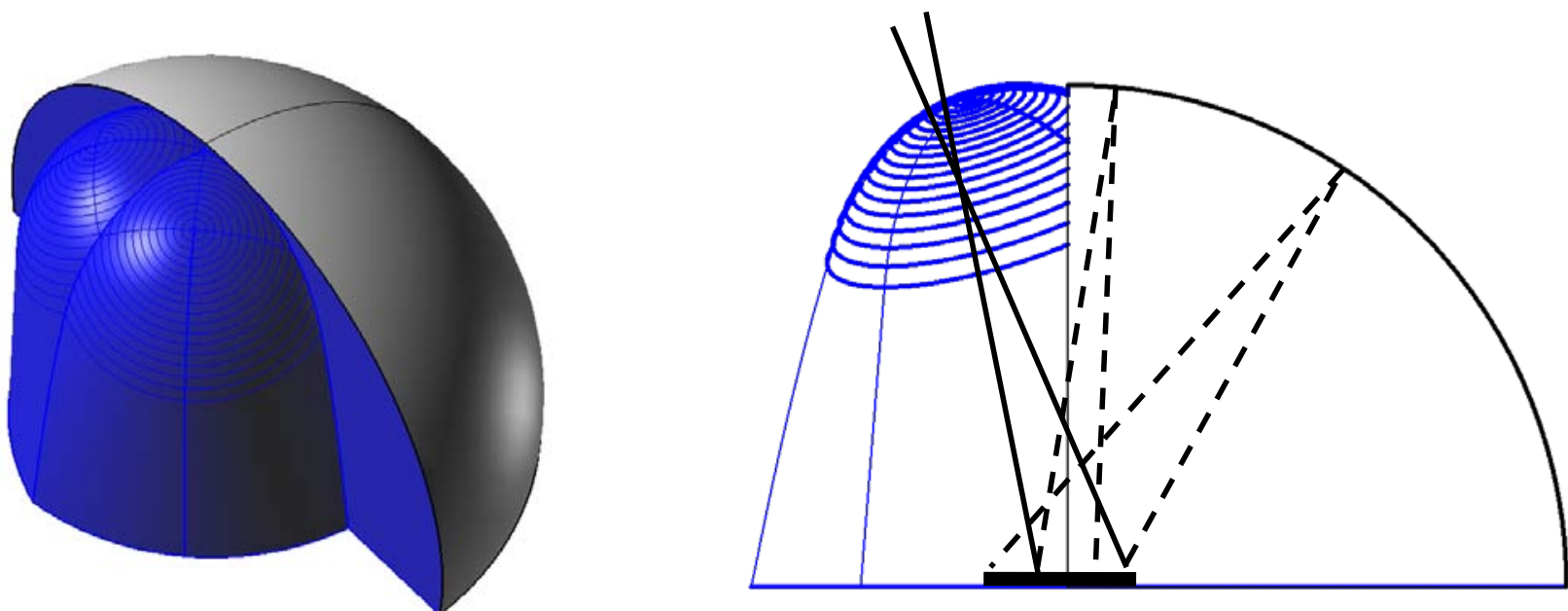

Figure 2 Left: FK-Cavity SOE 3D render, blue colored for the refractive parts and grey colored for the reflective confinement cavity. Right: SOE cross-section. Rays impinging on the cell (continuous lines) get reflected on the cell surface, on the bottom of the SOE. Reflected rays (dashed lines) hit the cavity surface, are reflected again and redirected towards the cell. 
For present multijunction cells, the light reflected on the semiconductor surface is specular (the cell surface roughness is very small, similar to good optical mirrors), while the light reflected on the grid lines has a significant diffuse component caused by the grid line geometry and roughness. However, it is remarkable that the roughness and imperfections of the grid line surface still keep the same cylindrical symmetry, as a good approximation. Therefore, the direction of the scattered light conserves the vector component along the grid line, and a light ray is scattered inside the surface of a cone (see Figure 1).

This is important for the design of the concentrator and the external cavity (which traditionally has assumed random Lambertian scattering): the illumination of the cell needs to come only be from one hemisphere, while the cavity occupies the other (asymmetric cavity). Figure 2 shows this asymmetric cavity concept in the particular case of being applied to an FK concentrator SOE. The figure shows how incident light rays (coming from the module primary element, POE) enter the refractive part of the SOE towards the cell, and the reflected part of this incoming light hits the metalized cavity and returns towards the cell again.

The 3D geometrical shape of the confinement cavity used for our module is a revolution symmetry ellipsoid (see Figure 3). Due to its symmetry, its design has been developed in 2D (we have chosen a cross-section). Focuses of the crosssection 2D ellipse are placed on a circle in the cell plane (black points in Figure 3), with its area equal to that of the solar cell (square-shaped). With this criterion we are ensuring we will almost recover the whole amount of reflected light. It would be possible to recover $100 \%$ of reflected light if cell was circular-shaped.

Figure 3 shows an example of confinement cavity (it is shown just a quarter of the whole cavity for illustration reasons and since in our concentrator we will use just a quarter of the whole ellipsoid). As can be seen in the figure, rays impinging on the design surface (blue) get reflected towards the cavity and get reflected again, impinging on the opposite point of the surface.

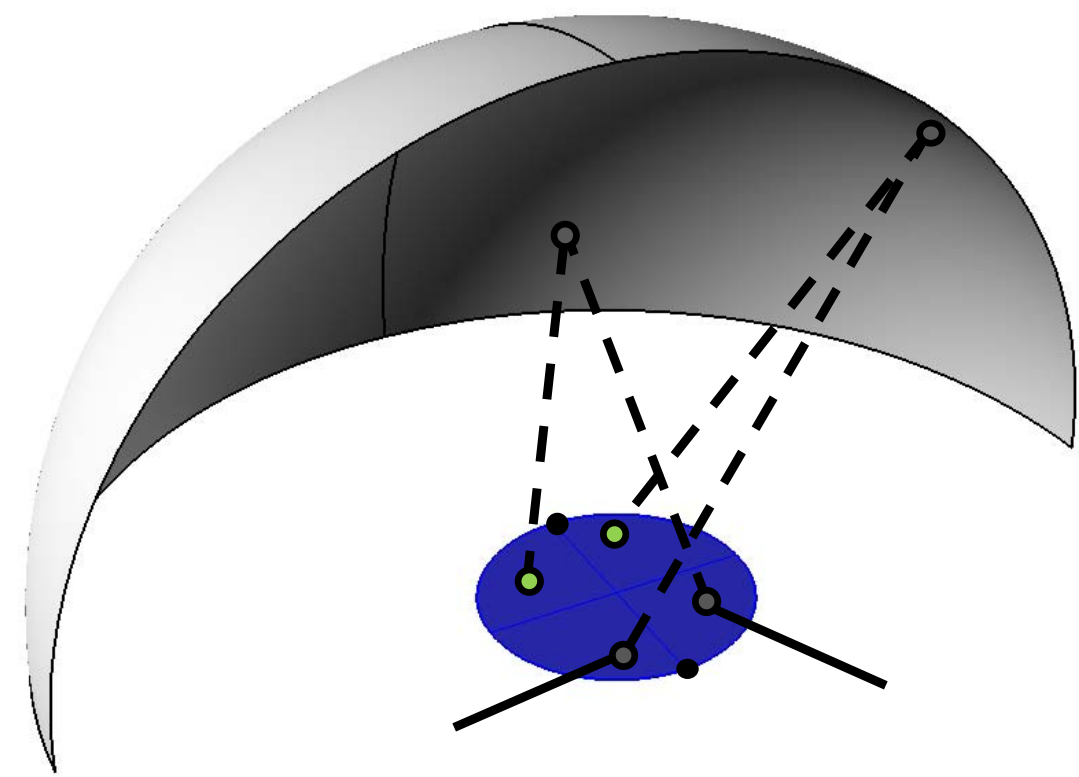

Figure 3 Example of cavity performance: cavity surface in grey color; cavity’s aim surface in blue; impinging rays in continuous lines; reflected rays in dashed lines; cavity design points in black; grey points denote rays reflections; green point for absorbed rays.

As shown in Figure 4 rays are focused by primary optical element (a Fresnel lens, like in FK concentrator) on the SOE. Since we are using an asymmetric cavity, for the SOE we are just using two of the original FK four sectors, and the remaining two sectors have been replaced by the cavity. In the same way, to focus light in those two SOE sectors, we just need two of the original POE four-sectors of the FK concentrator. 


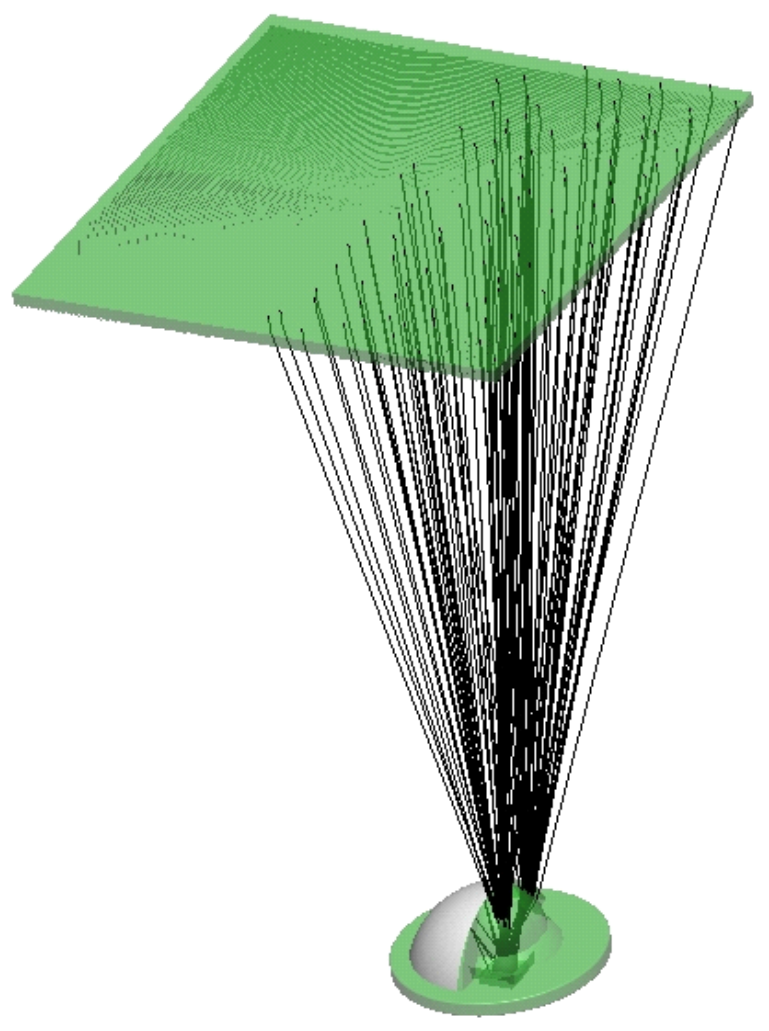

Figure 4 FK-Cavity render with raytrace simulation included. POE is placed in the upper part (only two of four parts of the original FK architecture are used). Some simulated rays impinging on the cell get reflected on its surface and are recovered by cavity.

\section{CAVITY EFFICIENCY GAIN MODEL}

We propose a mathematical analysis method to model the amount of light recovered by the external cavity (and so the efficiency gain achieved). The recovered portion of light will just depend on two parameters: shading factor $\left(f_{s}\right)$ and concentration $(C)$. We show results calculated for the FK-Cavity module (FK with cavity) for different values of $f_{s}$ and $C$ and we compare them with the same calculations made for the FK-NoCavity (original FK concentrator, without confinement cavity integration). For this comparison a PMMA POE, a B270 SOE and a 90\% reflectivity external cavity have been chosen for optical concentrator specifications, while for the cell we have used Spectrolab CDO-100-C3MJ manufacturer data.

These particular results are shown in Figure 5, where we offer cell electrical efficiency results with and without cavity (not taking into account losses introduced by the optical concentrator in both cases). In the two upper graphics different curves are plotted for different $f_{s}$ values, except the dashed one, which is the envelope curve of all the other curves (i.e. for each value of concentration it shows the result for the $f_{s}$ value which maximizes efficiency). It is simple to see and easy to understand looking at Figure 5 that, if we compare, the same $f_{s}$ value, and for a particular $C$, a FK-Cavity curve with a FK-NoCavity one, the first one presents always a higher efficiency than the latter. Besides, we can observe that the efficiency gain is higher at higher $f_{s}$ values in the FK-Cavity case.

This effect is mainly due to the important loss of absorbed light for FK-NoCavity case, when the cell surface covered by grid lines (i.e. $f_{s}$ ) is large. Moreover, FK-NoCavity presents especially poor efficiency results when dealing with $f_{s}$ values over $10 \%$, for the whole concentration range. However, for the same high $f_{s}$ values, FK-Cavity manages to recover an important part of light reflected by grid lines so efficiency values are outstanding. 

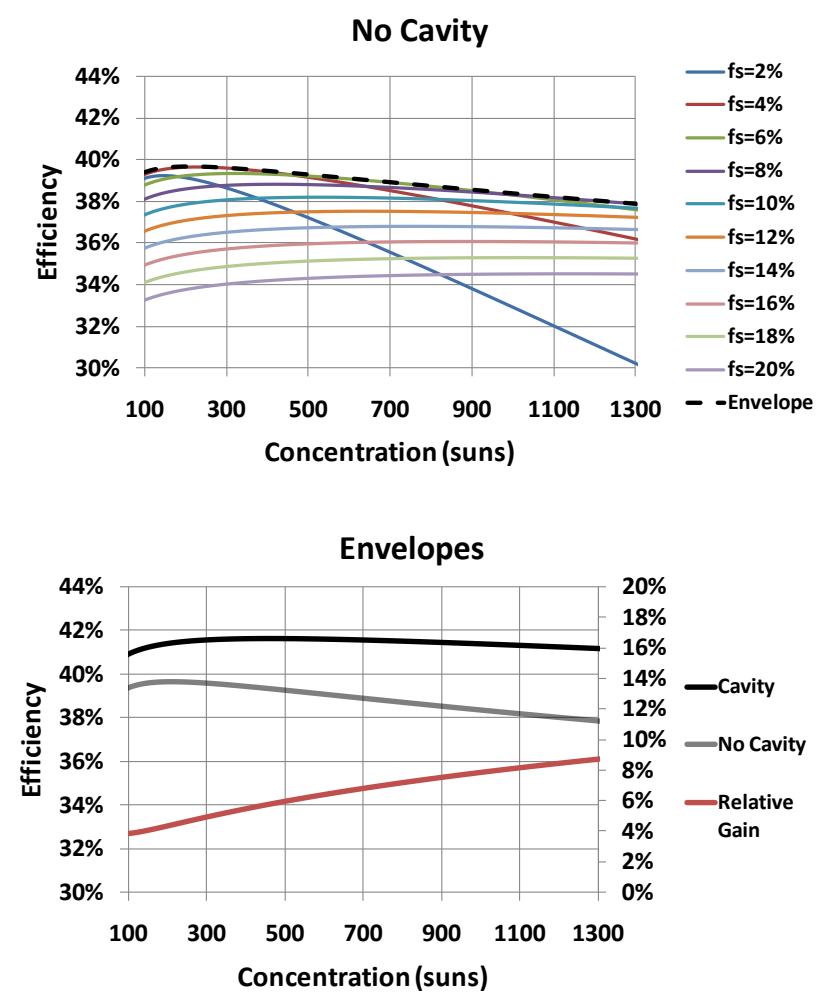
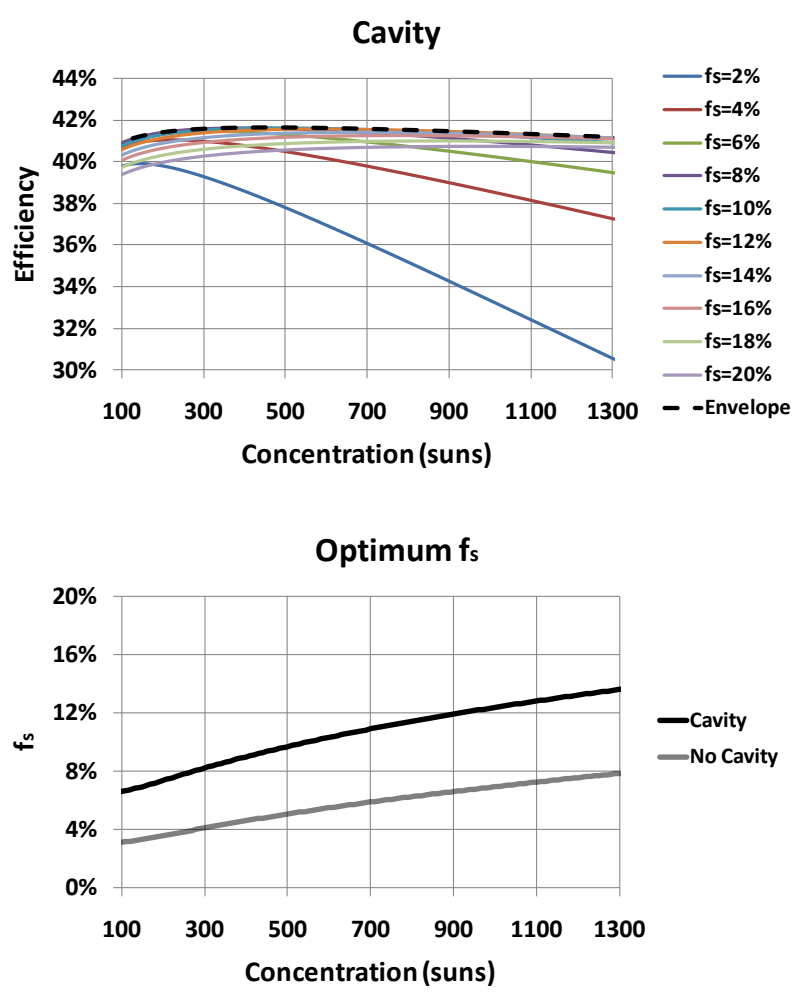

Figure 5 Top: graphics for No Cavity and Cavity modules simulating different concentration and shading factor values. Bottom left: comparison of envelope curves and relative gain. Bottom right: comparison for optimum values of shading factors.

Another extracted conclusion for these two graphics is that each value of $f_{s}$ has its optimum $C$, and the higher $f_{s}$ is the higher optimum $C$ value will be [7] (even if this optimum $C$ value is different for both modules). The explanation of this effect has to do with series resistance $\left(R_{s}\right)$. For a given $C, R_{s}$ is lower at higher $f_{s}$ values since we are dealing with higher grid lines area. This situation leads to a trade-off based on $f_{s}$ : low $f_{s}$ values increase semiconductor light absorption, but also increase $R_{s}$, while on the other hand, high $f_{s}$ values decrease semiconductor light absorption, but also decrease $R_{s}$.

Figure 5 (bottom-left graphic) shows the comparison of maximized efficiency FK-Cavity and FK-NoCavity modules for each level of concentration (i.e. comparison of the upper graphics' envelopes) and the relative gain of introducing the cavity concept in the FK architecture. The graphic shows in a very neat way the significant efficiency gain introduced by the confinement cavity concept, for any $C$ value. As shown in Figure $\mathbf{5}$ bottom-right graphic, $f_{s}$ optimum value strongly depends on concentration factor, as stated above. Since high $C$ values mean high optimum $f_{s}$ values, when dealing with high concentrations, FK-NoCavity will lose a great amount of light due to the high density of grid lines. This unabsorbed light will be recovered in the FK-Cavity case thanks to the cavity, so there will be a really significant efficiency difference between both modules when working at high $C$.

\section{MEASURED EFFICIENCY GAIN}

This chapter is focused on showing results obtained with our manufactured FK-Cavity module and their comparison with the results offered by an equivalent FK-NoCavity module. For these comparative measurements we have used the same housing, POE, solar cell and heatsink for both modules. Thus the only difference between both modules is the SOE (both without AR coating). For this purpose we have used two identical glass pieces (both SOEs with external cavity), metalizing with silver the cavity for the FK-cavity case (and protecting with paint) and just covering cavity with paint for the FK-NoCavity case (see Figure 6).

In the FK-Cavity case a paint layer has been applied over the metalized cavity surface in order to protect the metallization from oxidation. In the FK-NoCavity case paint prevents from Fresnel reflection due to the non-metalized 
cavity refractive surface. So we will have a 90\% (approximately) reflectivity cavity in the first case and a $0 \%$ reflectivity in the second one. Our two modules are working at a concentration of 170 suns, and chosen cell presents a $f_{s}=8 \%$.

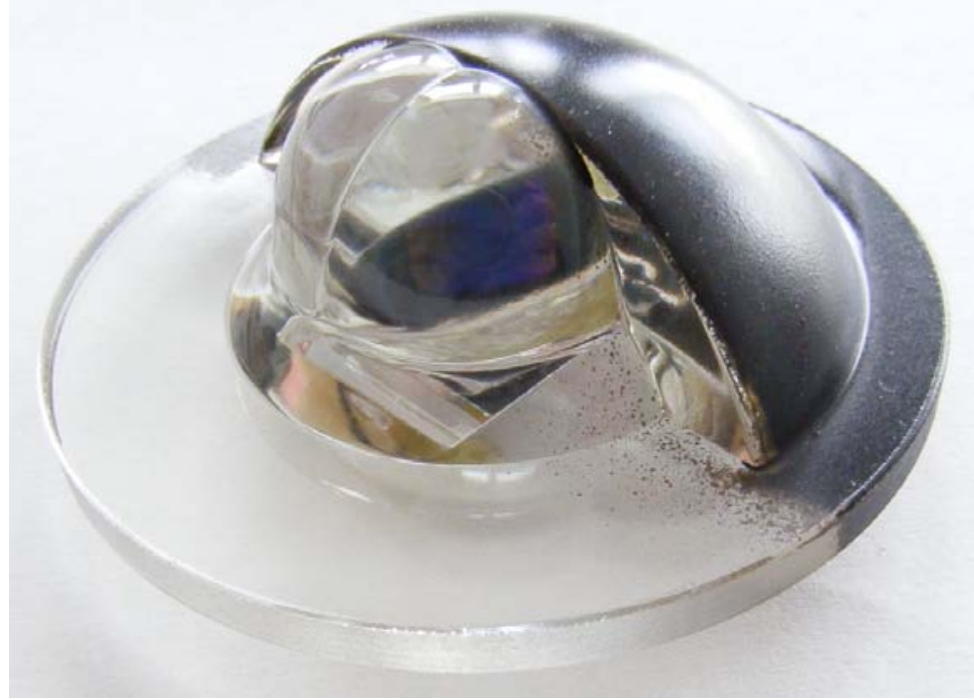

Figure 6 FK-Cavity SOE. The two original parts from FK concentrator remain uncovered while the cavity part is metalized and protected with a paint layer.

\subsection{Indoors measurements}

The indoors measurements have been made with a collimated source of white light. We have placed successively our two modules: FK-Cavity and FK-NoCavity. In this case we have compared short-circuit currents $\left(I_{s c}\right)$ between both modules, obtaining a very good result: $4.5 \%$ relative gain with the FK-Cavity module. Figure 7 shows the setup employed for the indoors measurements.

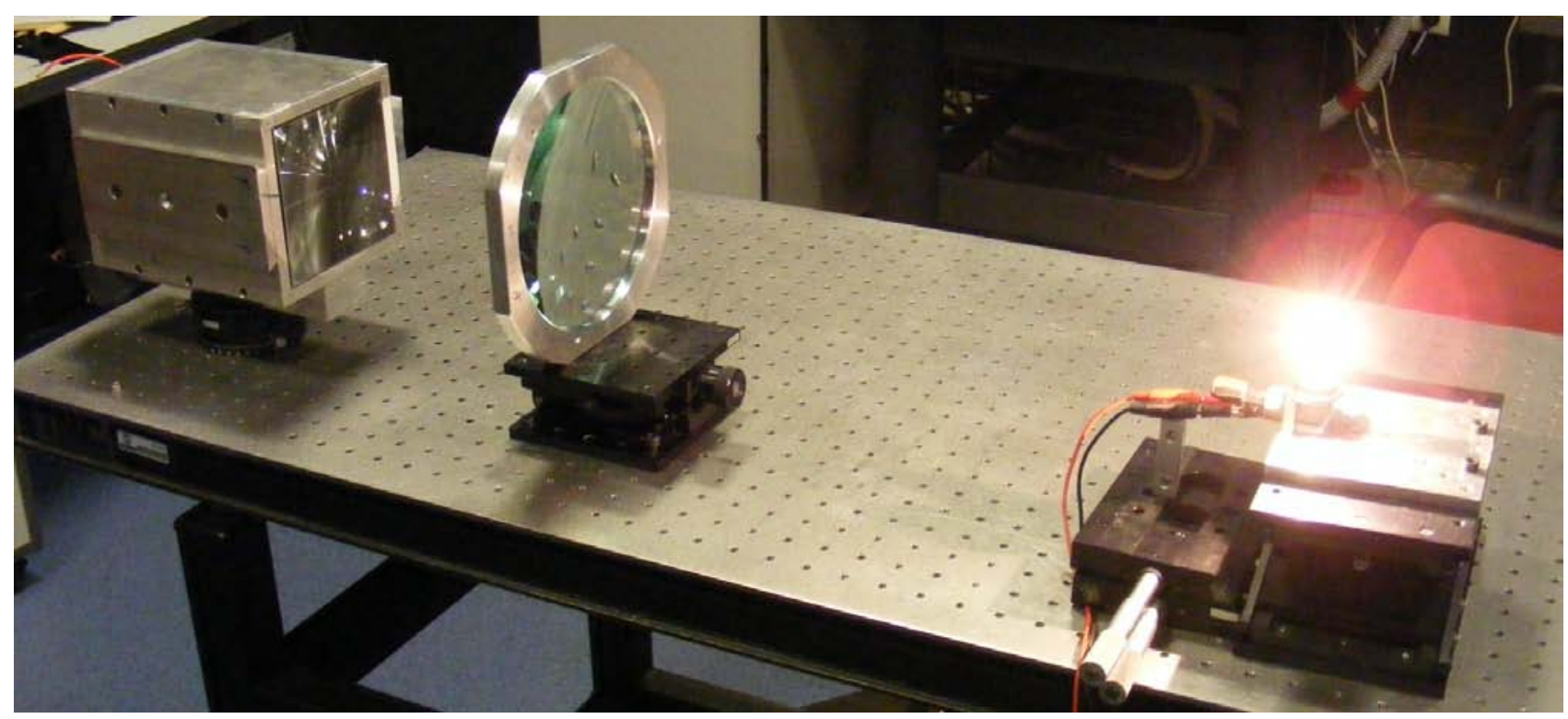

Figure 7 Setup for the indoors measurements. From right to left: omnidirectional white source, collimating lens, FK-Cavity module (entry aperture with the 4-sectors Fresnel lens can be seen). This setup provides a collimated white light at the module entry aperture. 


\subsection{Outoors measurements}

To make a fair comparison between both systems, we have measured them at the same moment of the day, so the solar spectrum is exactly the same and so the Jratio=Jtop/Jmiddle of the solar cell. This second system comparison has offered even better results than the indoors one. As shown in Figure 8, for the FK-Cavity we have a $I_{s c}=0.78 \mathrm{~A}$ and a module electrical efficiency $\eta=32.4 \%$ (33.2\% @ Tcell $=25^{\circ} \mathrm{C}$ ), while FK-NoCavity presents $I_{s c}=0.732 \mathrm{~A}$ and $\eta=30.6 \%$. This means outstanding results: a 6.0\% electrical efficiency relative gain and a 6.5\% Isc relative gain.

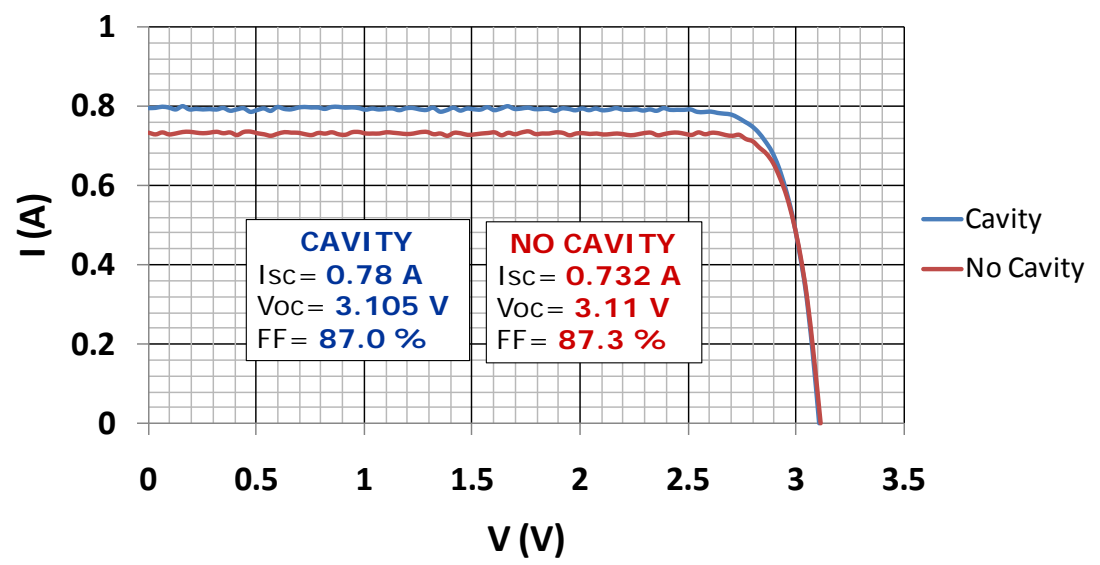

Figure 8 I-V curve comparison between FK-Cavity and FK-NoCavity

\section{CONCLUSIONS}

It is the first time to report the manufacturing of a CPV module prototype integrating an external confinement cavity. This cavity integration in a CPV module has been possible thanks to the use of LPI Fresnel-Köhler advanced concentrators. In the particular case of this publication, a high efficiency FK module has been used for the manufactured prototype. Excellent indoors and outdoors results have been obtained with this first prototype including the external cavity concept. This has a vital importance since it has been proven the great performance of this kind of cavities when included in CPV module architectures. As stated along the text these promising results encourages us to design more complex CPV designs also integrating external confinement cavities [4].

\section{ACKNOWLEDGEMENTS}

Authors thank the Spanish Ministries MCINN (ENGINEERING METAMATERIALS: CSD2008-00066, DEFFIO: TEC2008-03773, SIGMASOLES: PSS-440000-2009-30), MITYC (ECOLUX: TSI-020100-2010-1131, SEM: TSI020302-2010-65) and the Madrid Regional Government (SPIR: 50/20100.23/12/09,TIC2010 and O-PRO: PIE/209/2010) and UPM (Q090935C59) for the support given in the preparation of the present work. They also want to thank ISI group at the IES (Solar Energy Institute of Madrid), and especially S. Askins, for the help with indoors measurements.

\section{REFERENCES}

[1] Miñano, J. C., “Optical confinement in photovoltaics” in [Phisical Limitations to the Photovoltaic Solar Energy Conversion], Luque, A., Araujo, G. L., Adam Hilger, Bristol (1990).

[2] Benitez, P., et al., "High performance Fresnel-based photovoltaic concentrator" Opt. Express 18, A25-A40 (2010).

[3] Buljan, M., et al., "Improving performances of Fresnel CPV system: Fresnel-RXI Köhler concentrator”, Proc. 25th EU PVSEC, Valencia (2010). 
[4] Buljan, M., Benitez, P., Mohedano, R., Miñano, J. C., "Free-form Fresnel RXI-RR Köhler design for highconcentration photovoltaics with spectrum-splitting”, Proc. SPIE Optics + Photonics, San Diego (2011).

[5] O’Neill, U.S. Patent No. 4,711,972.

[6] Boca, A., et al., "Prismatic Covers for Boosting the Efficiency of High-Concentration PV Systems," Proc. 34 IEEE PVSC (2009).

[7] Algora, C., Diaz, V., "Influence of series resistance on guidelines for manufacture of concentrator p-on-n GaAs solar cells”, Prog. in Photovoltaics, 8, 211-225 (2000). 